\title{
Apparent diffusion coefficient as a potential surrogate marker for Ki-67 index in mucinous breast carcinoma.
}

\section{$\operatorname{AUTHOR}(S)$ :}

Onishi, Natsuko; Kanao, Shotaro; Kataoka, Masako; lima, Mami; Sakaguchi, Rena; Kawai, Makiko; Kataoka, Tatsuki R; Mikami, Yoshiki; Toi, Masakazu; Togashi, Kaori

\section{CITATION:}

Onishi, Natsuko ... [et al]. Apparent diffusion coefficient as a potential surrogate marker for $\mathrm{Ki}-67$ index in mucinous breast carcinoma.. Journal of magnetic resonance imaging 2014, 41(3): $610-615$

\section{ISSUE DATE:}

2014-03-04

URL:

http://hdl.handle.net/2433/200265

\section{RIGHT:}

This is the peer reviewed version of the following article: Onishi, N., Kanao, S., Kataoka, M., lima, M., Sakaguchi, R., Kawai, M., Kataoka, T. R., Mikami, Y., Toi, M. and Togashi, K. (2015), Apparent diffusion coefficient as a potential surrogate marker for Ki-67 index in mucinous breast carcinoma. J. Magn. Reson. Imaging, 41: 610-615, which has been published in final form at http://dx.doi.org/10.1002/jmri.24615. This article may be used for non-commercial purposes in accordance with Wiley Terms and Conditions for Self-Archiving.; This is not the published version. Please cite only the published version.; この論文は出版社版でありません。引用の際には出版社版をご確認ご利用ください。 


\section{Abbreviated Title Page}

Apparent diffusion coefficient as a potential surrogate marker for Ki-67 index in mucinous breast carcinoma 


\section{Abstract \\ Purpose}

To examine the association between apparent diffusion coefficient (ADC), cellularity and Ki-67 index in mucinous breast carcinoma (MBC) compared with invasive carcinoma of no special type (NST). ADC's ability to identify lesions with highly-proliferating MBC was also examined.

\section{Materials and Methods}

Pathologically confirmed MBCs (mucinous group, $\mathrm{n}=18$ ) and NSTs (control group, $\mathrm{n}=18$ ) were retrospectively analyzed. ADC was calculated from signal intensity of diffusion weighted imaging at $\mathrm{b}$ values of 0 and $1000 \mathrm{sec} / \mathrm{mm}^{2}$. Ki-67 index and cellularity were histopathologically evaluated. The mucinous group was classified into high Ki-67 mucinous group (Ki-67 index $\geq 14 \%$, highly-proliferating) and low Ki-67 mucinous group.

\section{Results}

In the mucinous group, minimum ADC (ADCmin) showed inverse correlation with cellularity ( $r=-0.802, \mathrm{p}=<0.0001)$ and Ki-67 index $(\mathrm{r}=-0.825, \mathrm{p}=<0.0001)$. In the control group, ADCmin showed inverse correlation with cellularity $(\mathrm{r}=-0.537 \mathrm{p}=0.022)$, but no correlation with Ki-67 index ( $\mathrm{r}=0.035$, $\mathrm{p}=0.892$ ). ADCmin of high Ki-67 mucinous group was significantly lower than that of low Ki-67 mucinous group $(\mathrm{p}=0.005)$.

\section{Conclusion}

This study demonstrated an inverse correlation between ADC and Ki-67 index in MBC and the ability of ADC to identify highly-proliferating MBC. Considering that ADC can evaluate whole lesions noninvasively, ADC may be a promising non-invasive surrogate marker for Ki-67 index in the risk stratification of MBC. 
Key words:

ADC, Mucinous carcinoma, Breast cancer, Cellularity, Ki-67 index 


\section{INTRODUCTION}

Diffusion weighted MR imaging (DWI) is well recognized as a useful non-contrast MR sequence for breast carcinoma(1). In various organs, including the breast, an inverse correlation between tumor cellularity and apparent diffusion coefficient (ADC; a quantitative parameter in DWI) is generally observed $(2,3)$. Several DWI studies have demonstrated significantly lower ADC for breast carcinomas than for benign breast lesions (2-4).

In the clinical practice of breast cancer management, risk stratification is as important as distinguishing between malignant and benign. Since uncontrolled proliferation is a key feature of the progression of malignancy, immunohistochemical assessment of the nuclear antigen Ki-67 is now widely used as a tool for breast cancer stratification $(5,6)$. The nuclear antigen Ki-67 is detectable in cells at all phases of the cell cycle except the G0 phase (7), and the Ki-67 index (the percentage of cells with Ki-67 positive nuclear immunostaining) is a measure of tumor proliferation. There is growing interest in the use of Ki-67 index as a pharmacodynamic biomarker of treatment efficacy in samples taken before, during, and after neoadjuvant therapy, particularly neoadjuvant endocrine therapy (6).

In previous reports $(8,9)$, neither invasive carcinomas (various types) nor invasive ductal carcinomas of not otherwise specified type (equivalent to "invasive carcinoma of no special type" in the newest WHO classification (10)) showed significant correlation between ADC and Ki-67 index. Considering the inverse correlation between ADC and tumor cellularity $(2,3)$, these results may imply that there is no correlation between cellularity and Ki-67 index in invasive carcinoma of no special type (NST).

In breast MR imaging, mucinous breast carcinoma (MBC) is dealt with differently from 
other types of breast carcinoma, because MBC presents higher ADC than benign breast lesions and other malignant breast tumors due to abundant mucin and low cellularity, as reported by Woodhams et al.(11). Better prognostic factors have been reported in pure MBC than in mixed MBC(12,13), and in hypocellular type pure MBC than in cellular type (14), which suggested an association between cellularity and prognostic factors in MBC. Considering these special features of MBC, we hypothesized that ADC is associated with Ki-67 index in MBC.

The aims of this study were 1) to examine the association between ADC, cellularity and Ki-67 index in MBC compared to luminal-type NST, and 2) to evaluate the potential of ADC to distinguish between highly proliferating and low proliferating MBC.

\section{MATERIALS AND METHODS}

\section{Patients}

Approval for this study was obtained from the Institutional Review Board of our institution, and informed consent was waived due to retrospective study design. Between September 2008 and March 2012, 17 females (mean age=58.6 years, age range=39-76 years) were pathologically diagnosed with mucinous breast carcinoma (hypocellular type of pure MBC, 11; cellular type of pure MBC, 5; mixed MBC, 1) at our institution. As a control group, 17 female patients (mean age $=60.5$ years, age range $=43-80$ years) diagnosed with luminal-type NST during the same period of time were selected, matched for histological grade, MR scan date (within two months) and MR scanner type (3.0 T/ 1.5 T). Every patient underwent breast MRI scan and pathological evaluation by core needle biopsy (CNB) or surgery at intervals of two months maximum. Patients receiving neoadjuvant endocrine therapy (NAE) or neoadjuvant chemotherapy (NAC) underwent 
MRI scans and pathological evaluations at the same period of therapies (pre- / post-).

Seventeen patients with MBC (14 without neoadjuvant therapy, 2 post NAE, 1 pre \& post NAE) provided 18 lesions (mucinous group: hypocellular type of pure MBC, 11; cellular type of pure MBC, 5; mixed MBC, 2). Seventeen patients with luminal-type NST (14 without neoadjuvant therapy, 2 post NAC, 1pre \& post NAC) provided 18 lesions (control group).

\section{MRI Acquisition}

Between September 2008 and March 2012, breast MRI was performed with a 3.0/1.5 T scanner (MAGNETOM Trio/Avant, A Tim System, Siemens AG) with 16/4ch breast coil. Routinely, T2-weighted, T1-weighted, Diffusion-weighted and fat-suppressed T1-weighted dynamic contrast enhancing images were obtained. Bilateral breast diffusion-weighted images were obtained using the following parameters: axial orientation, single shot-EPI, TR/TE 7000/62 ms, FOV 330×160 mm, matrix 166×80, thickness $3 \mathrm{~mm}$, NEX 3, 48 slices. Other parameters were as follows: T2-weighted images; 6000/85-87ms, thickness 2.5mm / T1-weighted images; 3D-Gradient echo 4.87/2.45, thickness $1.25 \mathrm{~mm}$ / fat-suppressed T1-weighted dynamic contrast enhancing images; pre- and post- (three times at $0-1,1-2$ and 5-6 min after gadolinium injection) contrast whole breast axial scanning in high temporal resolution for 1 min (3D-VIBE: R/TE 3.8/1.48 ms, FA 15 and FOV 330 mm×330 mm, matrix 448×461, 2.5 mm thickness, 60 slices), post contrast (at 2-5 min after gadolinium injection) whole breast coronal scanning in high-resolution (3D-VIBE: TR/TE 4.2/1.5 ms, FA 15, FOV $330 \mathrm{~mm} \times 330$ mm, matrix 448 $\times 412$, thickness $0.8 \mathrm{~mm}, 176$ slices). Infused gadolinium contrast materials were either Gadoteridol (ProHance, Eisai Inc., Tokyo, Japan) or Gadodiamide 
(Omniscan, Daiichi-Sankyo Inc., Tokyo, Japan) at a dose of $0.2 \mathrm{ml} / \mathrm{kg}$ power injected at a speed of $2.0 \mathrm{ml} / \mathrm{s}$ and flushed with $20 \mathrm{ml}$ of saline at the same rate.

\section{Image Analysis}

Breast carcinoma lesions were detected and analyzed by diffusion-weighted images in association with T2-weighted, T1-weighted and dynamic contrast enhancing images in order to accurately identify the lesions.

ADC values were calculated using the algorithm presented by the following equation: $A D C=\left[1 /\left(b_{2}-b_{1}\right)\right] \times \operatorname{In}\left[S_{1} / S_{2}\right]$, where $S_{1}$ and $S_{2}$ are the signal intensities in the region of interest (ROI) obtained by two gradient factors, $b_{1}$ and $b_{2}\left(b_{1}=0 \mathrm{sec} / \mathrm{mm}^{2}, b_{2}=1000\right.$ $\mathrm{sec} / \mathrm{mm}^{2}$ ). Inside the breast carcinoma lesions, placing as many $3 \times 3 \mathrm{~mm}$ circle ROIs as possible without overlapping areas, the average ADC (ADCave) and the minimum ADC (ADCmin) values were obtained. At this time, cystic or necrotic areas were carefully excluded by checking T2-\&T1-weighted and dynamic contrast enhancing images. These radiological evaluations were made by a breast radiologist (SK; 15 years of experience).

\section{Pathological Analysis}

Specimens were resected surgically and fixed overnight in $10 \%$ neutralized buffered formalin, then cut into $5 \mathrm{~mm}$ slices, and embedded in paraffin. Paraffin-embedded samples were serially sliced into $4 \mu \mathrm{m}$ slices, and deparaffined by xylene. Serial sections were stained with hematoxylin-eosin (HE), and immunostained using anti-estrogen receptor (ER) antibody and anti-Ki-67 antibody (DAKO Cytomation, Glostrup, Denmark). Pathological diagnoses were made by breast pathologists (TRK with 13 years of experience and YM with 24 years of experience) independently. 
The average of the estrogen receptor (ER) positivity was $95.7 \%$ (range $70-100 \%$ ) in the mucinous group and 98.3\% (range 90-100\%) in the control group.

\section{Ki-67 Index}

The Ki-67 index was assessed microscopically. All tumor cell nuclei with homogenous granular staining, multiple speckled staining, or nucleolar staining were considered positively stained regardless of intensity, while any cytoplasmic immunoreactivity was considered non-specific, and hence not taken into consideration. Scoring was performed in the areas with highest number of positive nuclei (hot spot) within the tumor. The Ki-67 index was expressed as the percentage of Ki-67 positive malignant cells in 1,000 malignant cells assessed under high power magnification $(\times 400)$.

In the context of the St. Gallen consensus 2013 (15), we classified the mucinous group into "low Ki-67 mucinous group (Ki-67 index; <14\%)" and "high Ki-67 mucinous group (Ki-67 index; $\geq 14 \%)$ ".

\section{Analysis of Tumor Cellularity}

Pathologists initially selected the areas with highest number of Ki-67 positive nuclei (hot spot), its adjacent area, and lowest number of Ki-67 positive nuclei (cold spot) in each specimen stained by Ki-67. At 20-fold magnification, these three fields of view (FOVs: 1.6 $\times 1.2 \mathrm{~mm}$ ) were digitally photographed and saved as image files. HE- and ER-stained slides were likewise photographed at the same FOVs. In this way, 9 image files were saved for each specimen. From these image files, the tumor cellularity was analyzed according to the methods of previous studies $(2,11)$.

All ER-stained images were converted to RGB color mode. In each image, some ER 
positive tumor cell nuclei were selected using the range selection tool, and their RGB thresholds were computed. Adjusting the RGB thresholds of each image according to the computed values, only ER-positive tumor cell nuclei were visualized. Each ER stained image was converted to black \& white mode and the binarized image was obtained. Using histogram, composition area ratio of ER positive tumor cell nuclei (=[counts of black pixels / total pixels]×100) was calculated. Multiplying this ratio by 100/ER positivity, tumor cellularity was calculated. Three values were calculated for each specimen from three FOVs. Considering the correspondence to Ki-67 index, the maximum value among the three was chosen as the tumor cellularity.

\section{Statistical Analysis}

Statistical analysis was performed with JMP10 (SAS institute). Correlation between ADCmin/ave, tumor cellularity and Ki-67 index was evaluated using Pearson's correlation coefficient. ADC values of the high Ki-67 mucinous group and low Ki-67 mucinous group were compared using Mann-Whitney test. A P value of less than 0.05 was considered statistically significant.

\section{RESULTS}

Correlation between ADCmin/ave, tumor cellularity and Ki-67 index is summarized in Table 1. In the mucinous group, ADCmin showed an inverse correlation with cellularity $(\mathrm{r}=-0.802, \mathrm{p}<0.0001)$ (Figure1a) and with Ki-67 index $(\mathrm{r}=-0.825, \mathrm{p}<0.0001)$ (Figure1b). Correlation between cellularity and Ki-67 index was also significant ( $\mathrm{r}=0.633, \mathrm{p}=0.005)$. In the control group, ADCmin showed an inverse correlation with cellularity ( $\mathrm{r}=-0.537$, $\mathrm{p}=0.022)$, but no significant correlation with Ki-67 index $(\mathrm{r}=0.035, \mathrm{p}=0.892)$. There was 
no significant correlation between cellularity and Ki-67 ( $r=0.032, p=0.899)$ in this group. ADCave showed correlation patterns similar to ADCmin in both the mucinous and control group, but correlations tended to be weaker with ADCave than with ADCmin (Table1).

When comparing the high and low Ki-67 mucinous groups, ADCmin of the high Ki-67 mucinous group was significantly lower than that of the low Ki-67 mucinous group ( $p=0.005$ ) (Figure2a). The high Ki-67 mucinous group can be identified with a sensitivity of $100 \%$ and a specificity of $93 \%$ by using the cut-off value of ADCmin $=1.27 \times 10^{-3} \mathrm{~mm}^{2}$ /sec.

Among three different types of MBC, the mixed MBC and the cellular type of pure MBC showed lower ADCmin than the hypocellular type of pure MBC (Figure2b).

Microphotographs of specimens (HE staining and Ki-67 immunohistochemical staining) from two representative MBC lesions, and the corresponding MR images (ADCmap), are shown in Figure3 and 4. The hypocellular type of pure MBC lesion shows low cellularity, low Ki-67 index and high ADCmin (Figure 3), while the mixed MBC lesion shows high cellularity, high Ki-67 index and low ADCmin (Figure 4).

\section{DISCUSSION}

We demonstrate an inverse correlation between ADC and Ki-67 index in MBC. The high Ki-67 mucinous group presented significantly lower ADCmin than the low Ki-67 mucinous group and can be classified with the cut-off value of ADCmin=1.27 $\times 10^{-3} \mathrm{~mm}^{2} / \mathrm{sec}$. These results suggest the potential of ADC as a non-invasive surrogate biomarker for Ki-67 index. It should be emphasized that ADC can be used to evaluate the whole lesion non-invasively, while Ki-67 index only evaluates a limited part of the lesion 
sampled by biopsy.

MBC is generally known for its relatively favorable prognosis, but MBC contains subtypes with aggressive progression and prognosis (16). Therefore, evaluating the cellular proliferative potential is crucial in the appropriate treatment of MBC. Ki-67 index is currently of interest as a pharmacodynamic biomarker of treatment efficacy in samples taken before, during, and after neoadjuvant therapy, particularly neoadjuvant endocrine therapy. Our results suggest that ADC can be used for this purpose in MBC.

In the control group, ADCmin showed an inverse correlation with cellularity, but no significant correlation with Ki-67 index. This is different from the results in the mucinous group, where ADCmin and cellularity showed significant correlation with Ki-67 index. These contrasting results may be explained by considering the relation between cellularity and Ki-67 index from a histopathological viewpoint. NST morphologically vary considerably and may contain extremely variable stromal components (e.g. foci of elastosis, focal necrosis) (10). As cellularity is affected by the ratio between the area for the tumor cells and the surrounding stroma, it is speculated that cellularity is affected by the presence of stromal components such as central necrosis or fibrosis (CNF). Considering that high Ki-67 index is associated with CNF in invasive ductal breast carcinoma (17) and lymph node-negative breast cancer (18), breast cancer with high Ki-67 index may not necessarily have high cellularity, which is compatible with the result in the control group where no correlation was seen between cellularity and Ki-67 index. In contrast, the correlation between cellularity and Ki-67 index seen in the mucinous group may imply that MBC, characterized by proliferation of nests of cells floating in lakes of mucin (16), has less stromal components that affect cellularity.

The association between subtypes, cellularity and prognostic factors in MBC has been 
previously described. MBC can be classified into 3 subtypes: hypocellular type of pure MBC (major type), cellular type of pure MBC, and mixed MBC (containing non-mucinous “cellular” components consisting of infiltrating carcinoma without extracellular mucin). Anan et al(13) reported that incidences of lymphatic vessel invasion and nodal involvement were lower in pure MBC than in mixed MBC. Fentiman et al(12) reported that pure MBC had significantly better relapse-free and overall survival than mixed MBC. These reports suggest the importance of differentiating mixed MBC from pure MBC. Also, differentiation of the cellular type of pure MBC from the hypocellular type may be important according to Clayton(14), who demonstrated good correlation between low cellularity and survival in pure MBC. In our study, the mixed MBC and cellular type of pure MBC presented significantly lower ADCmin than the hypocellular type of pure MBC. MBC has been excluded from several reported studies in the potential use of ADC for breast cancer detection because of its high ADC mimicking benign lesions (11,19). Our results suggest ADC may have the ability for detecting MBC with the poorer prognosis. If we can obtain the same result on larger sample size in the future, it might be helpful for the detection and risk stratification of MBC.

The above discussion further indicates a wider application of ADC as a biomarker of cell proliferation in additional situations. ADC is particularly associated with Ki-67 index in MBC because cellularity is associated with tumor aggressiveness (12-14). If we can identify the specific situations where cellularity is associated with cell proliferation (e.g. among specific subtype or during chemotherapy), ADC may be used as a biomarker of cell proliferation in such situations, not limited to MBC.

Another interesting result from the current study is that ADCmin is a better surrogate marker than ADCave for Ki-67 index in MBC. Although ADCmin and ADCave showed 
an inverse correlation with Ki-67 index in the mucinous group, ADCmin showed a stronger correlation with Ki-67 index than ADCave. Considering that Ki-67 index is calculated using the "hot spot" counts in the pathology specimen, it is reasonable that ADCmin, reflecting information from presumably the most "cellular" area, is a better marker for Ki-67 index than ADCave.

A few limitations of this study should be noted. Firstly, luminal-type NST lesions (average ER positivity 98.3\%) were selected as a control group because all lesions in the mucinous group were luminal-type (average ER positivity 95.7\%), and cellularity was evaluated using ER stained images. Therefore, these study results cannot be applied to HER2 type and triple negative type. Retrospective study design and small sample size are other limitations, though these were inevitable because of the low frequency of MBC.

In conclusion, our analysis demonstrated 1) the inverse correlation between ADC and Ki-67 index in MBC, and 2) the ability of ADC to classify highly proliferating MBC from those that are low proliferating. Considering the advantage of ADC in evaluating whole the lesion non-invasively, in contrast to Ki-67 index which only evaluates part of the lesion, ADC may be a promising non-invasive surrogate marker for Ki-67 index in the risk stratification of MBC. 


\section{REFERENCES}

1. Tsushima Y, Takahashi-Taketomi A, Endo K. Magnetic resonance (MR) differential diagnosis of breast tumors using apparent diffusion coefficient (ADC) on 1.5-T. J Magn Reson Imaging 2009;30(2):249-255.

2. Guo Y, Cai YQ, Cai ZL, et al. Differentiation of clinically benign and malignant breast lesions using diffusion-weighted imaging. J Magn Reson Imaging 2002;16(2):172-178.

3. Hatakenaka M, Soeda H, Yabuuchi H, et al. Apparent diffusion coefficients of breast tumors: clinical application. Magn Reson Med Sci 2008;7(1):23-29.

4. Woodhams R, Matsunaga K, Iwabuchi K, et al. Diffusion-weighted imaging of malignant breast tumors: the usefulness of apparent diffusion coefficient $(\mathrm{ADC})$ value and $\mathrm{ADC}$ map for the detection of malignant breast tumors and evaluation of cancer extension. J Comput Assist Tomogr 2005;29(5):644-649.

5. Urruticoechea A, Smith IE, Dowsett M. Proliferation marker Ki-67 in early breast cancer. J Clin Oncol 2005;23(28):7212-7220.

6. Dowsett M, Nielsen TO, A'Hern R, et al. Assessment of Ki67 in breast cancer: recommendations from the International Ki67 in Breast Cancer working group. J Natl Cancer Inst 2011;103(22):1656-1664.

7. Gerdes J, Lemke H, Baisch H, Wacker HH, Schwab U, Stein H. Cell cycle analysis of a cell proliferation-associated human nuclear antigen defined by the monoclonal antibody Ki-67. J Immunol 
1984;133(4):1710-1715.

8. Kim S, Cha E, Kim H, et al. Diffusion-weighted imaging of breast cancer: correlation of the apparent diffusion coefficient value with prognostic factors. J Magn Reson Imaging 2009;30(3):615-620.

9. Jeh SK, Kim SH, Kim HS, et al. Correlation of the apparent diffusion coefficient value and dynamic magnetic resonance imaging findings with prognostic factors in invasive ductal carcinoma. J Magn Reson Imaging 2011;33(1):102-109.

10. Ellis IO, Collins L, Ichihara S, MacGrogan G. Invasive caecinoma of no special type. In: Lakhani SR, Ellis IO, Schnitt SJ, et al. WHO Classification of Tumours of the Breast, Fourth Edition. Lyon: IARC: 2012. p 34-38.

11. Woodhams R, Kakita S, Hata H, et al. Diffusion`weighted imaging of mucinous carcinoma of the breast: evaluation of apparent diffusion coefficient and signal intensity in correlation with histologic findings. AJR Am J Roentgenol 2009;193(1):260-266.

12. Fentiman IS, Millis RR, Smith P, Ellul JP, Lampejo O. Mucoid breast carcinomas: histology and prognosis. $\mathrm{Br} \mathrm{J}$ Cancer 1997;75(7):1061-1065.

13. Anan K, Mitsuyama S, Tamae K, et al. Pathological features of mucinous carcinoma of the breast are favourable for breast-conserving therapy. Eur J Surg Oncol 2001;27(5):459-463.

14. Clayton F. Pure mucinous carcinomas of breast: morphologic features 
and prognostic correlates. Hum Pathol 1986;17(1):34-38.

15. Goldhirsch A, Winer EP, Coates AS, et al. Personalizing the treatment of women with early breast cancer: highlights of the St Gallen International Expert Consensus on the Primary Therapy of Early Breast Cancer 2013. Ann Oncol 2013;24(9):2206-2223.

16. Bussolati G, Sapino A. Mucinous carcinoma and carcinomas with signet-ring-cell differentiation. In: Lakhani SR, Ellis IO, Schnitt SJ, et al. WHO Classification of Tumours of the Breast, Fourth Edition. Lyon: IARC: 2012. p 60-61.

17. Hasebe T, Sasaki S, Imoto S, Ochiai A. Highly proliferative fibroblasts forming fibrotic focus govern metastasis of invasive ductal carcinoma of the breast. Mod Pathol 2001;14(4):325-337.

18. Maiorano E, Regan MM, Viale G, et al. Prognostic and predictive impact of central necrosis and fibrosis in early breast cancer: results from two International Breast Cancer Study Group randomized trials of chemoendocrine adjuvant therapy. Breast Cancer Res Treat 2010;121(1):211-218.

19. Tozaki M, Hoshi K. 1H MR spectroscopy of invasive ductal carcinoma: correlations with FDG PET and histologic prognostic factors. AJR Am J Roentgenol 2010;194(5):1384-1390. 


\section{Table1}

Correlation between ADCmin/ave, cellularity and Ki-67 index in mucinous group and control group; ADCave shows correlation patterns similar to ADCmin, but with larger p-values and weaker correlation than ADCmin

\begin{tabular}{lrrrr}
\hline & \multicolumn{2}{c}{ ADCmin } & \multicolumn{2}{c}{ ADCave } \\
& r & P-value & r & P-value \\
\hline $\begin{array}{l}\text { Mucinous group } \\
\text { ADC vs. Cellularity }\end{array}$ & -0.802 & $<0.0001$ & -0.788 & 0.0001 \\
ADC vs. Ki-67 index & -0.825 & $<0.0001$ & -0.695 & 0.001 \\
Cellularity vs. Ki-67 index & 0.633 & 0.005 & 0.633 & 0.005 \\
Control group & & & & \\
ADC vs. Cellularity & -0.537 & 0.022 & -0.412 & 0.090 \\
ADC vs. Ki-67 index & 0.035 & 0.892 & 0.005 & 0.985 \\
Cellularity vs. Ki-67 index & 0.032 & 0.899 & 0.032 & 0.899 \\
\hline $\begin{array}{l}\text { ADC; apparent diffusion coefficient } \\
\text { ADCmin; the minimum ADC }\end{array}$ & & & & \\
ADCave; the average ADC \\
r; Pearson's correlation coefficient
\end{tabular}




\section{Figure Legends}

\section{Figure1}

Correlations between ADCmin, cellularity and Ki-67 index in mucinous group

a). An inverse correlation is indicated between ADCmin and cellularity ( $r=-0.802$, $\mathrm{p}<0.0001)$.

b). An inverse correlation is indicated between ADCmin and Ki-67 index $(r=-0.825$, $\mathrm{p}<0.0001)$.

\section{Figure2}

Distribution of ADCmin in mucinous group

a). High Ki-67 mucinous group (Ki-67 index; $\geq 14 \%$ ) shows significantly lower ADCmin than low Ki-67 mucinous group ( $\mathrm{p}=0.005$; Mann-Whitney test).

b). Mixed MBC and cellular type of pure MBC show lower ADCmin than hypocellular type of pure MBC.

\section{Figure3}

Representative case of pure MBC (hypocellular type)

a). HE-stained specimen $(\times 40)$; shows clusters of carcinoma cells floating in lakes of mucin, representing hypocellular nature.

b). Ki-67 immunohistochemical stained specimen $(\times 40)$; shows nuclei of tumor cells positively stained (brown) (Ki-67 index=7.4\%).

c). Corresponding MR image (ADC map); shows lobulated mass with high ADC $\left(\right.$ ADCmin $\left.=1.90 \times 10^{-3} \mathrm{~mm}^{2} / \mathrm{sec}\right)$. 


\section{Figure4}

Representative case of mixed MBC

a). HE-stained specimen ( $\times 40)$; shows large clusters of cells along with mucus reflecting higher cellularity.

b). Ki-67 immunohistochemical-stained specimen ( $\times 40)$; shows relatively large number of positively-stained nuclei (brown) (Ki-67 index=34.7\%).

c). Corresponding MR image (ADC map); shows round mass with low ADC $\left(\mathrm{ADCmin}=0.88 \times 10^{-3} \mathrm{~mm}^{2} / \mathrm{sec}\right)$. 
a)

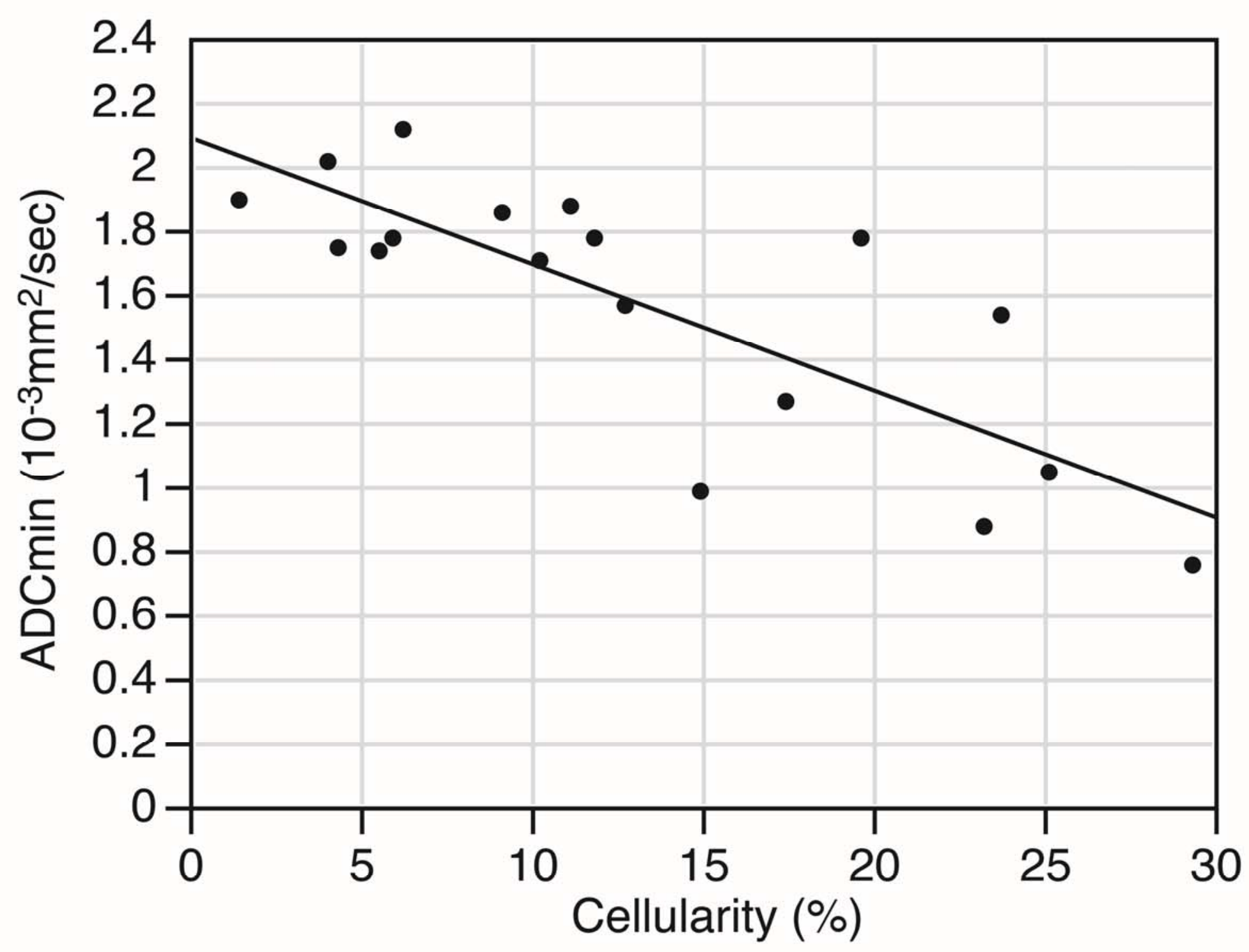

Figure 1 
b)

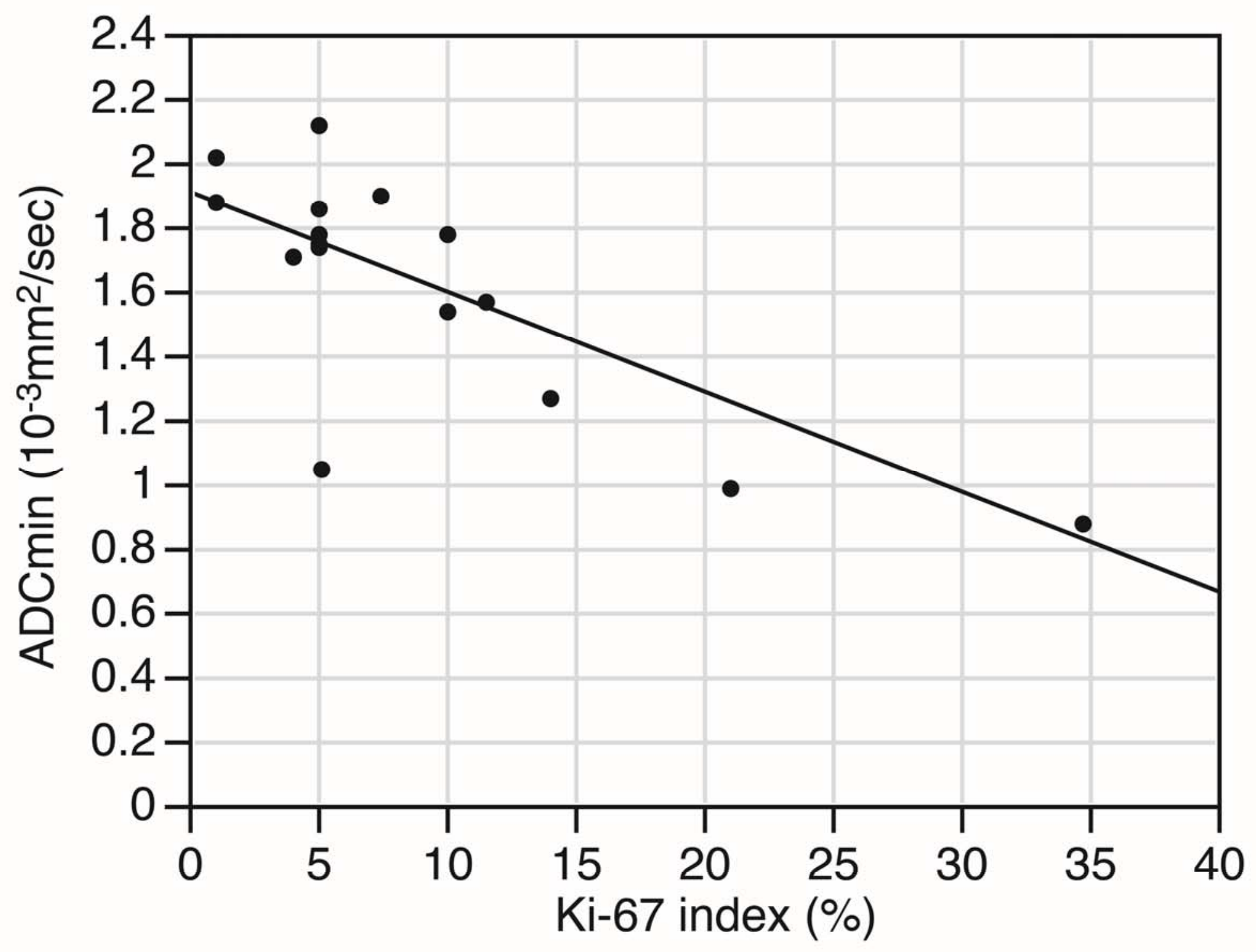

Figure 1 
a)

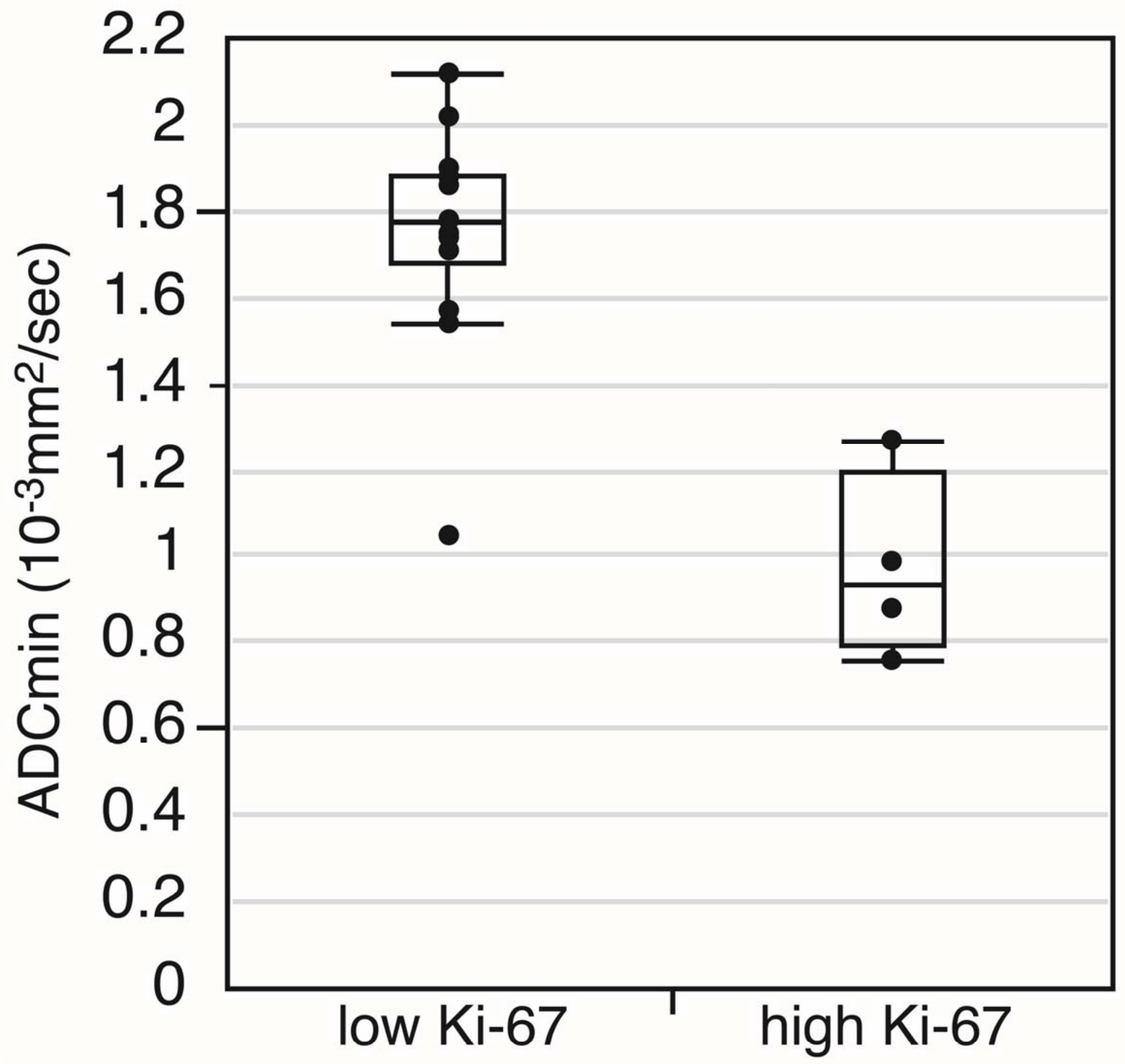
mucinous group mucinous group

Figure 2 
b)

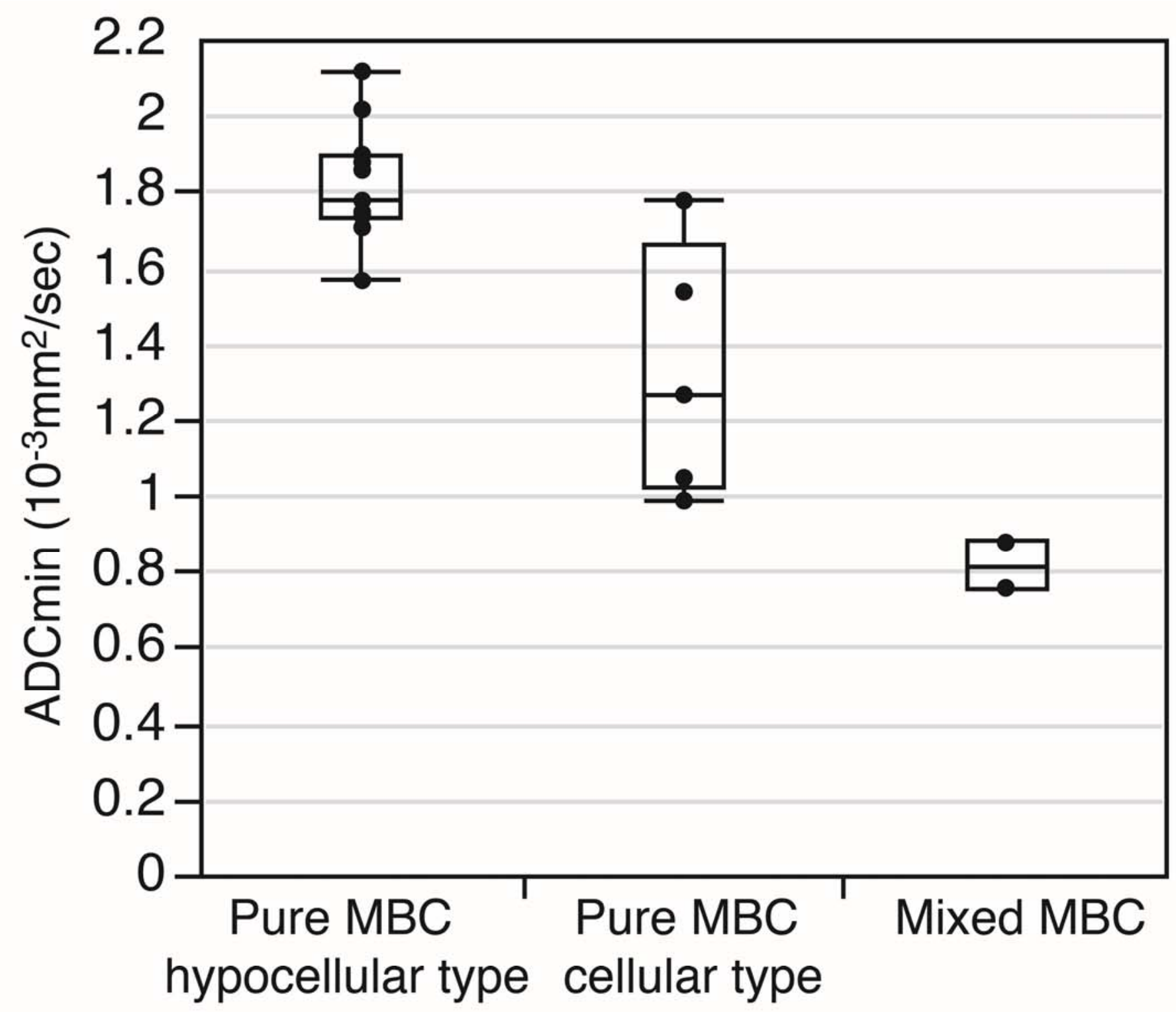

Figure 2 


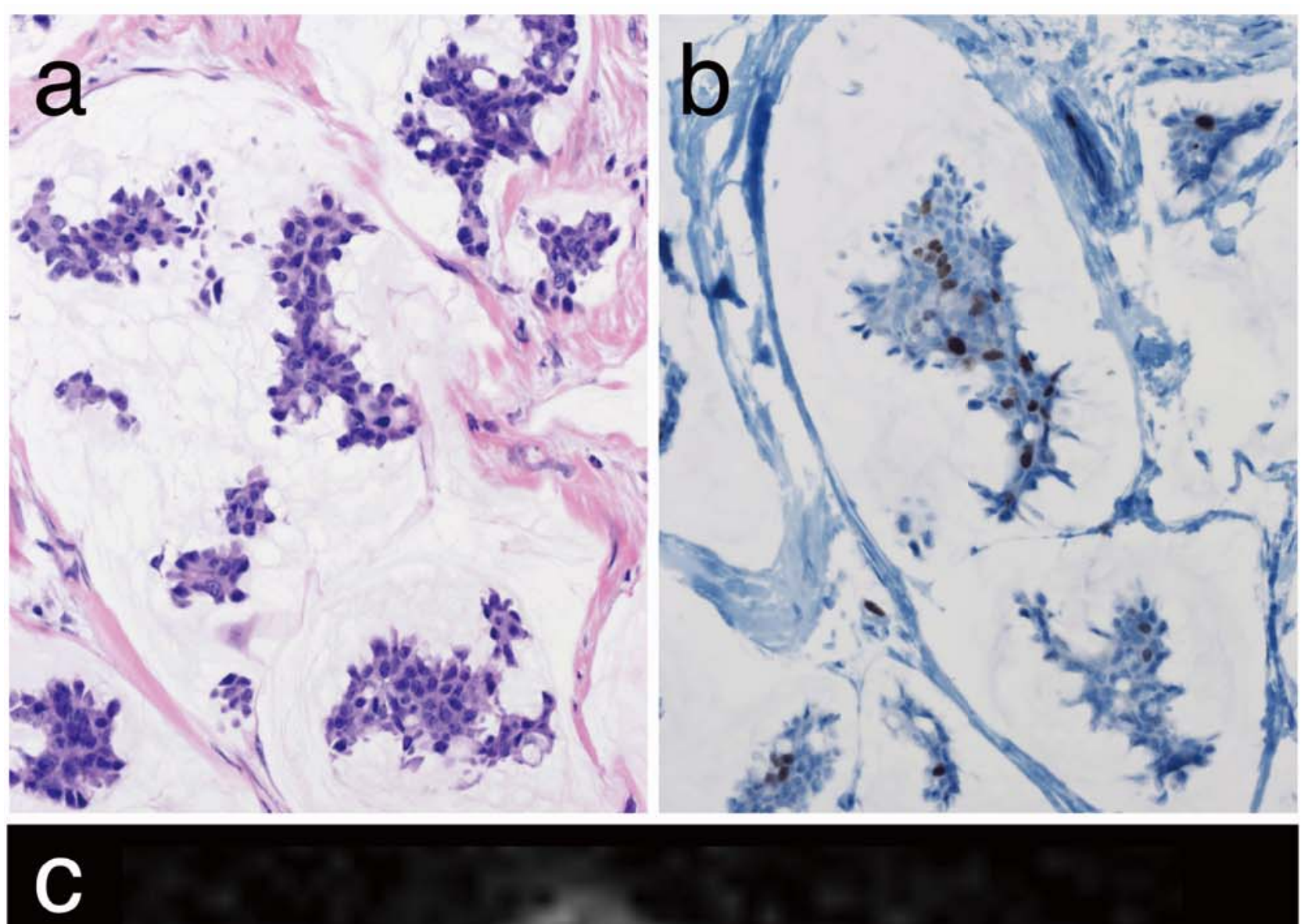

Figure 3 


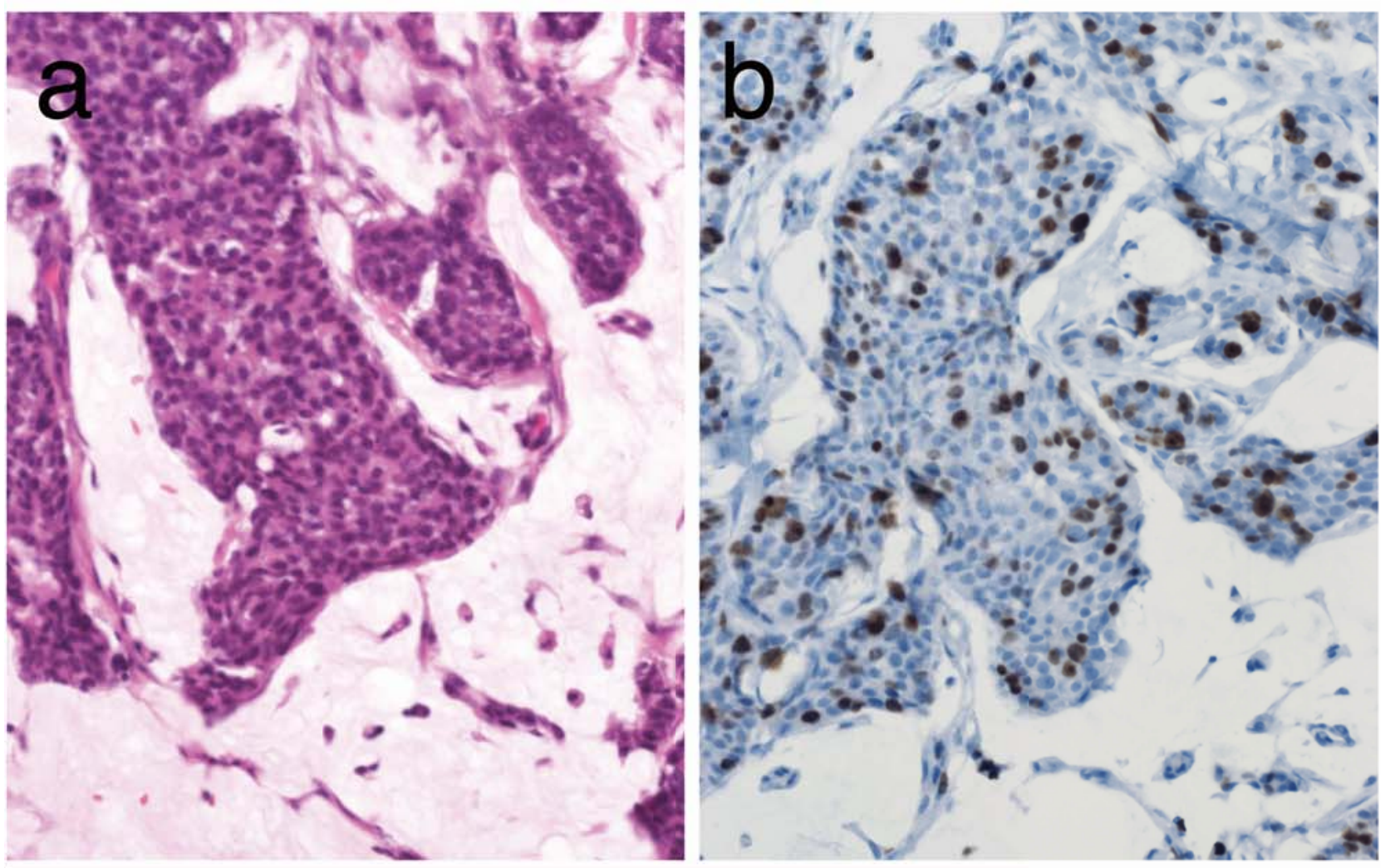

C 